hepth/9510227

UCSBTH-95-30

NSF-ITP-95-136

\title{
New Vacua for Type II String Theory
}

\author{
Joseph Polchinski \\ Institute for Theoretical Physics, University of California, \\ Santa Barbara, CA 93106-4030 \\ and \\ Andrew Strominger \\ Department of Physics, University of California, \\ Santa Barbara, CA 93106-9530
}

\begin{abstract}
Lorentz-invariant expectation values for antisymmetric tensor field strengths in Calabi-Yau compactification of IIA string theory are considered. These are found to impart magnetic and/or electric charges to the dilaton hypermultiplet. This results in a potential which can have supersymmetric minima at zero coupling or at conifold points in the moduli space. The latter occurs whenever the dilaton charge is aligned with that of the light black hole at the conifold. It is shown that there is a flat direction extending from the conifold along which there is a black hole condensate whose strength is of order the string coupling $g_{s}$. It is speculated that these new vacua correspond to string compactification on generalized Calabi-Yau spaces which have $c_{1}=0$ but are not Kahler.
\end{abstract}




\section{IIA COMPACTIFICATION WITH RAMOND-RAMOND BACKGROUNDS}

Consider IIA string theory compactified to four dimensions on a Calabi-Yau space $X$. The low-energy effective field theory contains $h_{11}(X)$ vector multiplets with moduli space $M_{V}$ (which includes the radial mode) and $h_{21}(X)+1$ neutral hypermultiplets (which includes the dilaton).

In ten dimensions the IIA theory contains 2-form $(G)$, 4-form $(F)$, and 10-form $(E)$ (as recently discovered in [1]) field strengths, as well as their 8-form, 6-form, and 0-form duals. In this paper we consider the effects of expectation values for these fields. An expectation value for the 10 -form $E$

$$
\langle E\rangle=\nu_{0} \epsilon^{(10)}
$$

where $\nu_{0}$ is a constant and $\epsilon^{(10)}$ is the 10-dimensional volume element, leads [1] to the massive 10-dimensional IIA theory with a cosmological constant equal to $\frac{1}{2} \nu_{0}^{2}$ discovered by Romans [2]. In the context of Calabi-Yau compactification, there are $2 h_{11}+1$ additional possibilities consistent with four-dimensional Lorentz invariance. The field strength $G$ may acquire an expectation value proportional to a harmonic form $\omega_{I}^{(2)}$ on $X$

$$
\langle G\rangle=\nu_{2}^{i} \omega_{i}^{(2)} \quad i=1, \cdots, h_{11}
$$

Similarly $F$ may acquire an expectation value proportional to a harmonic 4 -form on $X$ or to the 4-dimensional spacetime volume element $\mathcal{E}^{(4)}$

$$
\langle F\rangle=\nu_{4}^{i} \omega_{i}^{(4)}+\nu_{6} \epsilon^{(4)} \quad i=1, \cdots, h_{11} .
$$

Supersymmetry requires that all the $\nu$ 's vanish in a Calabi-Yau vacuum [3]. Since at the classical level the $\nu$ 's can be continuously adjusted to zero, their effects can be summarized in a supersymmetric, 4-dimensional effective action in which the $\nu$ 's appear as coupling constants. Since non-zero values of $\nu$ generically break supersymmetry,, we expect a modulidependent scalar potential $\mathcal{V}$.

\footnotetext{
${ }^{1}$ Quantization of the $\nu$ 's will be discussed below.
} 
Let us first consider the case where all the $\nu$ 's except $\nu_{0}$ vanish. The resulting 4dimensional theory is then obtained by compactification of the massive IIA theory in 10 dimensions. This latter theory contains a coupling, in the 10-dimensional Einstein frame,

$$
\frac{\nu_{0}}{2} \int d^{10} x \sqrt{-g} e^{3 \hat{\phi} / 2} G^{M N} B_{N M} \quad M, N=0,1, \cdots, 9
$$

where $B$ is the NS-NS two form potential and the 10-dimensional string coupling is $g_{s}=e^{\hat{\phi}}$. The reduction of this includes, in the four dimensional Einstein frame,

$$
\frac{\nu_{0}}{2} \int d^{4} x \sqrt{-g} e^{3 D} G^{\mu \nu} B_{\mu \nu} \quad \mu, \nu=0,1, \cdots, 3,
$$

where the scalar $D$ is related to the string frame volume $V$ of the Calabi-Yau by

$$
e^{3 D}=V .
$$

The field $D$ is an element of a vector multiplet.

The four-dimensional dilaton $\phi$ is part of a neutral hypermultiplet and is given by

$$
\phi=\hat{\phi}-\frac{3 D}{2} .
$$

The other three scalars in the hypermultiplet are the NS-NS axion $a$ defined by

$$
d a=e^{-4 \phi} * d B
$$

together with the two R-R axions parameterizing the expectation values of the 3-form potentials proportional to holomorphic and anti-holomorphic 3-forms on $X$.

To express (5) in a manner compatible with four-dimensional supersymmetry, we define a magnetic potential for $G$

$$
e^{3 D} * G=d \tilde{A}
$$

Integrating by parts, (5) then becomes

$$
\nu_{0} \int d^{4} x \sqrt{-g} e^{4 \phi} \tilde{A}_{\mu} \nabla^{\mu} a .
$$

This appears to violate (magnetic) gauge invariance, under which

$$
\tilde{A}_{\mu} \rightarrow \tilde{A}_{\mu}+\partial_{\mu} \epsilon .
$$


However, further couplings complete (10) to the perfect square

$$
\frac{1}{2} \int d^{4} x \sqrt{-g} e^{4 \phi} g^{\mu \nu}\left(\nabla_{\mu} a+\nu_{0} \tilde{A}_{\mu}\right)\left(\nabla_{\nu} a+\nu_{0} \tilde{A}_{\nu}\right) .
$$

Gauge invariance is then restored by accompanying (11) with the shift

$$
a \rightarrow a-\epsilon \nu_{0} .
$$

The complex scalar

$$
S=e^{-2 \phi}+i a
$$

which appears in an $N=1$ superfield can then be used to construct an operator $e^{\lambda S}$ which transforms as

$$
e^{\lambda S} \rightarrow e^{-i \nu_{0} \lambda \epsilon} e^{\lambda S}
$$

Thus this operator carries magnetic charge under the $U(1)$ Ramond-Ramond gauge symmetry of the IIA string. When $e^{\lambda S}$ has a nonzero expectation value, this implies that particles carrying the corresponding electric charge are confined. This can also be seen from the fact that the operator (5) gives mass to the gauge field and to $B_{\mu \nu}$. Noting that the $B_{\mu \nu}$ kinetic term is proportional to $e^{-2 \phi}$ and that the gauge field kinetic term is not, this mass is of order $e^{\phi}$ times the string scale.

One may also consider the effects of the other types of expectation values. It is not hard to see that for general values the dilaton acquires magnetic charges $\left(\nu_{0}, \nu_{2}^{i}\right)$ and electric charges $\left(\nu_{6}, \nu_{4}^{j}\right)$. Thus, by turning on R-R backgrounds arbitrary electric and magnetic charges may be imparted to the dilaton. NS-NS backgrounds also lead to charges.

There is also a mirror description of this phenomena in the context of IIB string theory. In that case there are two 3-form field strengths (one NS-NS and one R-R) which can acquire expectation values proportional to harmonic 3-forms on the Calabi-Yau.

\section{QUANTIZATION OF THE FIELD STRENGTH}

The fact that $e^{\lambda S}$ gets a magnetic charge proportional to $\nu_{0}$ strongly suggests that the latter is quantized. Ramond-Ramond charge is believed to be quantized in a unit which 
was denoted $\mu_{0}$ in ref. [1]. Then the corresponding magnetic charge is quantized in units of $2 \pi / \mu_{0}$, and so $\epsilon \sim \epsilon+\mu_{0}$ and the gauge transformation implies that

$$
a \sim a+\nu_{0} \mu_{0}
$$

However, $a$ was already periodically identified because of the shift encountered in encircling a fundamental string

$$
a \sim a+\frac{1}{2 \pi \alpha^{\prime}}
$$

These identifications must be commensurate, so $\nu_{0}$ is quantized. If, as seems likely, the identifications are the same, then

$$
\nu_{0} \mu_{0}=\frac{1}{2 \pi \alpha^{\prime}}
$$

Using the calculation of the charge quanta from ref. [1], this implies that $\nu_{0}$ is exactly $\mu_{8}$, the charge carried by the 8-brane. In other words, all values of $\nu_{0}$ that are allowed by the quantization are dynamically accessible by nucleation of 8-branes.

It is interesting to derive the same result directly in ten dimensions. Consider an 8-sphere surrounding a 0-brane which carries Ramond-Ramond charge. Integrating the field equation

$$
d *\left(e^{-2 \phi} H / 2\right)=\nu_{0} *\left(G+\nu_{0} B\right)
$$

over the 8-sphere seems to imply that $0=\nu_{0} \int *\left(G+\nu_{0} B\right)$, so the total flux is zero if $\nu_{0} \neq 0$. This would say that such charge is not observable at any scale, a result which is surprising because we have seen in the preceding section that the confinement scale is somewhat below the string scale. Suppose, however, that a fundamental string ends on the 0-brane, a possibility suggested by the identification of the latter as a D-brane. This adds a source $\left(2 \pi \alpha^{\prime}\right)^{-1}$ times a delta-function to the field equation, and since the string passes through the 8 -sphere once we now have $0=\nu_{0} \int *\left(G+\nu_{0} B\right)+\left(2 \pi \alpha^{\prime}\right)^{-1}$, giving the same quantization found above.

\footnotetext{
${ }^{2}$ We have normalized the $\mathrm{R}-\mathrm{R}$ fields to have a canonical kinetic term $\left(\alpha_{p}=1\right.$ in the notation of ref. [1]), and the NS-NS field $B_{\mu \nu}$ such that its coupling to the fundamental string is $\left(2 \pi \alpha^{\prime}\right)^{-1} \int B$.
} 
Thus, we have an interesting physical picture. Suppose that $\nu_{0}=n \mu_{8}$, and that there is a 0-brane/anti-0-brane pair each with a single unit of charge. Then the G-flux runs between them in a tube of width $g_{s}^{-1}$ times the string scale, and in addition they are connected by $n$ fundamental strings.

It is possible that this confinement will play an interesting role in string phenomenology, for example by removing states from the spectrum.

\section{THE SCALAR POTENTIAL AT LARGE RADIUS}

The scalar potential $\mathcal{V}$ in four dimensions is determined by the charges of the hypermultiplets and by $N=2$ supersymmetry. The general form of the potential is given for the case of electric charges in refs. [4,5]. This can be applied to the present (magnetically charged) case by simply performing a symplectic transformation which trades $G$ for $* G$ and turns the magnetic charge into an electric one [6]. The electric formula is

$$
\mathcal{V}=h_{u v} k_{I}^{u} k_{J}^{v} \bar{X}^{I} X^{J} e^{K}+\left(U^{I J}-3 \bar{X}^{I} X^{J} e^{K}\right) \mathcal{P}_{I}^{i} \mathcal{P}_{J}^{i}
$$

On the vector multiplet moduli space $M_{V}, X^{I}$ for $I=0,1, \cdots, h_{11}$ are complex projective coordinates, $K$ is the Kahler potential, which is related to a choice of holomorphic sections $\left(X^{I}, F_{I}\right)$ by $K=-\ln i\left(\bar{X}^{I} F_{I}-X^{I} \bar{F}_{I}\right)$, and

$$
U^{I J}=e^{K}\left(\partial_{a}+\partial_{a} K\right) X^{I} g^{a \bar{b}}\left(\partial_{\bar{b}}+\partial_{\bar{b}} K\right) \bar{X}^{J}
$$

with $a, b$ (non-projective) tangent space indices. On the hypermultiplet moduli space $M_{H}$, $h_{u v}$ is the metric, $k_{I}^{u}$ is the Killing vector which generates the action of the gauge transformation for the $I^{\prime}$ th abelian gauge field, and $\mathcal{P}_{I}^{i}$ for $i=1,2,3$ are an $S U(2)$ triplet of Killing potentials.

We will be concerned with the case that only one charge, which shall be labeled " 0 ," is non-vanishing. The non-zero components of $k$ follow simply from (13) as

$$
k_{0}^{u} \frac{\partial}{\partial u}=\nu_{0} \frac{\partial}{\partial a} .
$$

In this section the only relevant hypermultiplet is that containing the dilaton. The corresponding quaternionic geometry is obtained from ref. [0] by taking the special case $n=0$. In 
terms of the $N=1$ dilaton superfield $S$ and a superfield $C$ for the R-R sector components, the moduli space is Kahler with $K_{H}=-\ln 2\left(S+\bar{S}-[C+\bar{C}]^{2}\right)$. The norm of $k$ is then

$$
k_{0}^{u} k_{0}^{v} h_{u v}=\frac{\nu_{0}^{2}}{4} e^{4 \phi}
$$

where, for nonzero $C$, we define the dilaton by $2 e^{-2 \phi}=S+\bar{S}-[C+\bar{C}]^{2}$. Define

$$
u=e^{\phi} d C, \quad v=e^{2 \phi}[d S / 2-(C+\bar{C}) d C],
$$

Let $e=(u, v)$. Then the form $\Omega^{i}$ [4] constructed from the triplet of complex structures, and the corresponding potential $\omega^{i}$, are found to be

$$
\begin{aligned}
& \Omega^{i}=i e^{\dagger} \sigma^{i} e \\
& \omega^{1}=i(\bar{u}-u), \quad \omega^{2}=(\bar{u}+u), \quad \omega^{3}=i(v-\bar{v}) / 2
\end{aligned}
$$

These are related by

$$
d \Omega^{i}+\epsilon^{i j k} \omega^{j} \wedge \Omega^{k}=0
$$

The Killing potentials ( $D$-terms) are derived from the relation

$$
\mathbf{i}_{k_{I}} \Omega^{i}=-d \mathcal{P}_{I}^{i}-\epsilon^{i j k} \omega^{j} \mathcal{P}_{I}^{k}
$$

(i $\mathbf{i}_{k}$ denoting contraction of a form with a vector). The result is that the only nonvanishing component is

$$
\mathcal{P}_{0}^{3}=-\frac{1}{2} \nu_{0} e^{2 \phi}
$$

At large volume the only relevant vector multiplet is that containing the radial mode and corresponding axion, $t=b+i e^{D}$. The usual choice of sections is

$$
\widetilde{\Pi}=\left(\begin{array}{c}
\widetilde{F}_{0} \\
\widetilde{F}_{1} \\
\widetilde{X}^{0} \\
\widetilde{X}^{1}
\end{array}\right) \stackrel{D \rightarrow \infty}{=}\left(\begin{array}{c}
5 t^{3} / 6 \\
-5 t^{2} / 2 \\
1 \\
t
\end{array}\right),
$$

but because the 0 charge is magnetic we must make a symplectic transformation to 


$$
\widetilde{\Pi}^{\prime}=\left(\begin{array}{c}
\widetilde{F}_{0}^{\prime} \\
\widetilde{F}_{1}^{\prime} \\
\widetilde{X}^{\prime 0} \\
\widetilde{X}^{\prime 1}
\end{array}\right)=\left(\begin{array}{c}
\widetilde{X}_{0} \\
\widetilde{F}_{1} \\
-\widetilde{F}_{0} \\
\widetilde{X}^{1}
\end{array}\right) .
$$

in order to apply the formulae of [6]. One then finds that all terms in the potential (20) scale as

$$
\mathcal{V} \stackrel{D \rightarrow \infty}{\sim} \nu_{0}^{2} e^{4 \phi+3 D}
$$

in agreement with the potential derived by direct reduction of the 10-dimensional cosmological constant in the massive IIA theory. Similarly, an electric charge gives a potential $\sim \nu_{6}^{2} e^{4 \phi-3 D}$, again in agreement with that found directly from the action for $F$.

\section{IV. $N=1$ SUPERSYMMETRY}

Although we are considering here a compactification with $N=2$ spacetime supersymmetry, it is worth noting that the same phenomena will arise in $N=1$ compactifications, and the potential is easily derived using $N=1$ superfields. The difference from the familiar heterotic string compactifications is that the function $f$ multiplying the gauge field kinetic terms is now independent of the dilaton, since the gauge fields are in the $\mathrm{R}-\mathrm{R}$ sector. For the gauge field which descends from the 10-dimensional gauge field, the kinetic term is proportional to the volume of the compactified space, so $f \sim t^{3}$. The Kahler potential for the dilaton is the usual $-\ln (S+\bar{S})$. When the dilaton is charged the $D$-terms give a potential

$$
\mathcal{V}=\frac{1}{\operatorname{Im}(f)} \delta K \bar{\delta} K
$$

where $\delta$ and $\bar{\delta}$ refer to taking gauge variations of the chiral superfields and their conjugates respectively. An electric charge on the dilaton gives rise to a potential $S^{-2} t^{-3}$. For a magnetic charge we must first make a symplectic transformation to $f \propto t^{-3}$, and so the potential is $S^{-2} t^{3}$. These are the same as found above.

Related mechanisms were discussed in [8]. That paper considered the heterotic string, which has the important difference that a charge on the dilaton leads to an anomalous 
variation of the action, from the dependence of $f$ on $S$. Thus the charges carried by the dilaton are fixed so as to cancel the $U(1)$ anomalies from light fields. In the type II string the charge on the dilaton is not constrained by the anomaly. Also considered in refs. [9,8] were similar charges on moduli fields. These are not constrained by the anomaly, but they cannot appear in the low energy theory of the heterotic string because they lead to a potential for the moduli which is of order the string scale [9,8]. By contrast, the effect of the R-R field strengths can be regarded as a perturbation. This can be seen from the fact that the cosmological constant vanishes as the coupling is taken to zero in either the string or Einstein frame.

It will be interesting to find the heterotic string duals of our type II backgrounds. We have not yet explored this issue, except to note that the background $\nu_{6}$ becomes under six-dimensional string-string duality a magnetic field in the 4-5 direction. This heterotic background has been considered in the interesting recent work [10] and was also one motivation for ref. [11. It corresponds to giving a charge to a modulus [9, 8].

\section{THE SCALAR POTENTIAL NEAR A CONIFOLD}

Typically, as in (31), the potential will drive the theory to zero coupling which is uninteresting, or towards strong coupling, where non-perturbative effects must be considered.'? Life becomes much more interesting when one includes the effects of the charged BPS black holes (which can also be represented as D-branes [1]). In the IIA theory these arise from 0-, 2-, 4-, and 6-branes which wrap minimal, supersymmetric cycles in $X$. These can become massless at conifold points in the moduli space where the (world-sheet-instanton-corrected) periods degenerate. One then finds that the potential has flat directions corresponding to new supersymmetric string vacua.

To understand this in detail, let us consider the specific example of the IIA theory on the quintic $P_{4}(5)$ with $\nu_{0} \neq 0$ so that the dilaton carries magnetic charge. This theory has a

\footnotetext{
${ }^{3}$ However, we should note the recent result [12 that electric and magnetic field strengths in different $U(1)$ 's can break $N=2$ supersymmetry to $N=1$.
} 
single vector multiplet $\left(h_{11}=1\right)$ which includes the radial mode $D$. The moduli space $M_{V}$ has a conifold singularity [13] at which a single BPS black hole degenerates to zero mass [14]. It turns out that this black hole corresponds to a 6-brane wrapping the entire Calabi-Yau and carries the same magnetic charge as the dilaton. To see this we note that the geometry of $M_{V}$ is usually described by a period vector

$$
\Pi=\left(\begin{array}{c}
F_{0} \\
F_{1} \\
X^{0} \\
X^{1}
\end{array}\right)
$$

adapted to the mirror of $P_{4}(5)$ in which the entries are 3-cycle periods of the holomorphic 3 -form $\Omega$. A conifold singularity occurs at a point when $X^{1}=0$ and $F_{1}\left(X^{1}\right) \sim \frac{1}{2 \pi i} X^{1} \ell n X^{1}$. This is related to the earlier basis (29) by a symplectic transformation (for some choice of Kahler gauge for $\Pi$ ) which was determined in [13] as

$$
\widetilde{\Pi}=N \Pi
$$

where

$$
N=\left(\begin{array}{cccc}
0 & 0 & 0 & 1 \\
-1 & 0 & 0 & 0 \\
0 & -1 & 0 & 0 \\
-2 & 0 & -1 & 0
\end{array}\right)
$$

Hence if the period $X^{1}$ of $\Omega$ on the mirror of $P_{4}(5)$ is degenerating, the period $\widetilde{F}_{0}$, which approaches the volume at large radius, is also degenerating. It may sound rather strange that the entire 6-volume of $P_{4}(5)$ degenerates (while other cycles remain finite), but this is in a region where worldsheet instanton corrections are large and the distinction between 0-, 2-,4-, and 6-cycles is lost.

In ten dimensions, the 6 -brane acts as a source for the 8 -form $* G$. Hence a 6 -brane which wraps the Calabi-Yau carries the magnetic charge associated to $G$, just like a dilaton which acquires charge due to nonzero $\nu_{0}$. In four dimensions this leads to a charged hypermultiplet which we will represent by an $S U(2)$ doublet $B$. 
Next, let us consider the dilaton potential on the moduli space $M_{V} \otimes M_{H}$, ignoring for a moment the field $B$. It is convenient to work in the $\Pi$ basis (33), in which the dilaton carries electric charge in the "1" direction. The nonzero component of the Killing vector is then

$$
k_{1}^{u} \frac{\partial}{\partial u}=\nu_{0} \frac{\partial}{\partial a} .
$$

Near the conifold at $X^{1}=0, g_{1 \overline{1}}$ diverges as

$$
g_{1 \overline{1}} \sim-\ln \left(\bar{X}^{1} X^{1}\right)
$$

It follows that near the conifold, $\mathcal{V}$ has a local minimum

$$
\mathcal{V} \sim-\frac{\nu_{0}^{2}}{\ln \left(\bar{X}^{1} X^{1}\right)} .
$$

Hence, as pointed out in [14], the effective field theory without the black hole field $B$ can be used to show that the moduli are attracted to the conifold.

Near the conifold, the $B$ field gets light and must be included in the effective field theory. To leading order in $B$ and $e^{\phi}$, (36) becomes

$$
k_{1}^{u} \frac{\partial}{\partial u}=\nu_{0} \frac{\partial}{\partial a}+B \frac{\partial}{\partial B}-B^{\dagger} \frac{\partial}{\partial B^{\dagger}}
$$

where $B$ is a complex doublet. The Killing potential is

$$
\mathcal{P}_{1}^{i}=-\frac{\nu_{0}}{2} e^{2 \phi} \delta^{i 3}+B^{\dagger} \sigma^{i} B .
$$

The metric $g$ is now non-singular, the singularity in (37) having arisen from integrating out $B$ [14]. The first term in the potential (20) vanishes at the conifold point $X^{1}=0$, and the potential has a flat direction with unbroken $N=2$ supersymmetry, characterized by

$$
\mathcal{P}_{1}^{i}=0 .
$$

The vacua are parameterized by a single hypermultiplet which is a linear combination of $\Phi$ and $B$. At generic points the string coupling is non-zero and there is a black hole condensate. The vector modulus acquires a mass and is frozen at the conifold.

An important feature of these new vacua is that the expectation value of the black hole field is of order $g_{s}=e^{\phi}$. By taking $g_{s}$ to be small we can stay within the validity of both string 
perturbation theory and the expansion of the black hole hypermultiplet geometry (known only to leading order in $B$ ) about the conifold. The origin of this happy circumstance is ultimately the fact that the R-R fields appear with an extra factor of $g_{s}$ in the supersymmetry transformation laws.

In 115] it was argued that in some cases branches of the vacuum moduli space with black hole condensates were a dual description of known Calabi-Yau compactifications. This is unlikely to be the case here since this compactification has no massless vector multiplets and so cannot be a IIA Calabi-Yau compactification. A more likely possibility is that it corresponds to string compactification on one of the generalized (non-Kahler) Calabi-Yau spaces obtained by resolutions of small 3-cycles as 2-cycles. Such spaces are discussed for example in [16,13]. They are complex manifolds with $c_{1}=0$ but are in general not Kahler.

There are a number of extensions of our observations. Of particular interest is the possibility of breaking $N=2$ to $N=1$ along the lines recently described in [12]. This appears to require simultaneous expectation values for both R-R and NS-NS field strengths. Dilaton charges may also lead to new $N=4$ compactifications.

\section{ACKNOWLEDGMENTS}

We are grateful to K. Becker, M. Becker, S. Ferrara, J. Harvey, D. Lowe, S.T. Yau, and especially G. Moore for useful conversations. A recent attempt to generate a $D$ term in $N=2$ theories has been made by J. Harvey and G. Moore (unpublished). This work was supported in part by the Department of Energy, grant \#91ER40618 and NSF grants PHY91-16964 and PHY94-07194. 


\section{REFERENCES}

[1] J. Polchinski, Dirichlet Branes and Ramond-Ramond Charges. preprint NSF-ITP-95122, hepth/9510017 (1995).

[2] L. Romans, Phys. Lett., B169, 374, (1986).

[3] P. Candelas and D. Raine, Nucl. Phys., B248, 415, (1984).

[4] R. d'Auria, S. Ferrara, and P. Fre, Nucl. Phys., B359, 705 (1991).

[5] B. deWit, P. Lauwers, and A. van Proeyen, Nucl. Phys., B255, 569 (1985).

[6] A. Ceresole, R. D’Auria, S. Ferrara, and A. Van Proeyen, Nucl. Phys. B444, 92 (1995).

[7] S. Ferrara and S. Sabharwal, Nucl. Phys. B332, 317 (1990).

[8] M. Dine, N. Seiberg, and E. Witten, Nucl. Phys. B289, 589 (1987).

[9] M. Dine, N. Seiberg, X.G. Wen and E. Witten, Nucl. Phys. B289, 319 (1987).

[10] C. Bachas, A Way to Break Supersymmetry, preprint CPTH-R349-0395, hepth/9503030 (1995).

[11] S. B. Giddings, J. Polchinski, and A. Strominger, Phys. Rev. D48, 5784 (1993).

[12] S. Ferrara, L. Girardello, M. Porrati, preprint NYU-TH-95-10-02, hep-th/9510074 (1995).

[13] P. Candelas, L. Parkes, P. Green, and X. de la Ossa, Nucl. Phys. B359, 21 (1991).

[14] A. Strominger, Nucl. Phys., B451, 96 (1995); hep-th/9504090.

[15] B. Greene, D. Morrison, and A. Strominger, Nucl. Phys., B451, 109 (1995).

[16] M. Reid, Math. Ann., 274, 329 (1987). 